\title{
Clinical dermatoscopical response of glycyrrhizinic acid $0.1 \%$ spray in treating external anogenital warts in HIV patients: a case series
}

\author{
Pati Aji Achdiat, Rasmia Rowawi, Hendra Gunawan, Oki Suwarsa, Catherina Jessica Sutantoyo
}

Department of Dermatology and Venereology, Faculty of Medicine, Universitas Padjadjaran - Dr. Hasan Sadikin Hospital, Bandung, Indonesia

\begin{abstract}
Patients with human immunodeficiency virus (HIV) disease are more likely to develop anogenital warts (AGW) compared to HIV-negative people, and are susceptible to treatment failures and illness recurrences. Glycyrrhizinic acid (GA) is extracted from licorice root (Glycyrrhiza glabra). Previous studies have demonstrated the efficacy of GA $0.1 \%$ spray as a treatment for external AGW, but its role in immunocompromised patients has not been explored. The current study reported two cases of HIV-positive patients with AGW who experienced partial remission using GA $0.1 \%$ spray. The treatment was applied by the patients three times a day for eight weeks. Treatment evaluation were made by using visual inspection and dermoscopy image analysis. After twelve weeks, clinical observation revealed poor improvement with only slight reduction in number and size of the lesions; although dermoscopy findings revealed an evolution from finger-like to knob-like pattern. GA facilitate the development of T helper lymphocytes in cell-mediated immune response, whereas in HIV, there are a decreased number of $\mathrm{T}$ helper lymphocytes. This may explain poor therapeutic response in our patient. Dermoscope is useful in the evaluation of treatment progress, especially when invisible to unaided eye.
\end{abstract}

HIV AIDS Rev 2021; 20, 1: 65-69 DOI: https://doi.org/10.5114/hivar.2021.105050

Key words: anogenital warts, human papillomavirus, human immunodeficiency virus, glycyrrhizinic acid.

\section{Introduction}

Anogenital wart (AGW) is one of the most common sexually transmitted diseases (STD) in the world. The global annual occurrences of all types of AGWs range from 160 to 289 per 100,000 population [1]. An increased rate of human papillomavirus (HPV) infection was observed in human immunodeficiency virus (HIV)-infected patients. A host's immunocompetence has a significant role in the progression

Address for correspondence: Dr. Pati Aji Achdiat, Department of Dermatology and Venereology,

Faculty of Medicine, Universitas Padjadjaran - Dr. Hasan Sadikin

Hospital, Bandung, Indonesia, e-mail: anamorphic83@gmail.com

of the disease. HPV infection manifestations are more severe and prolonged among immunocompromised patients [2, 3].

AGW is benign proliferations of skin and mucosa caused by HPV infection. Those HPVs fall into two groups: "lowrisk" strains (HPV 6 and 11) that cause AGW and "high-risk" (oncogenic) strains (HPV 16 and 18), which cause anogenital cancers [4]. External AGW lesions are located on the penis, vulva, scrotum, perineum, and perianal. They vary in appearance, manifesting in papular wart, condyloma acumina-

Article history:

Received: 13.04.2019

Received in revised form: 02.09.2019

Accepted: 20.08.2020

Available online: 30.03 .2021
International Journal of HIV-Related Problems

HIV \& AIDS

R e vi e w 
ta (cauliflower-like masses), keratotic warts, and flat-topped papules [5]. The diagnosis of AGW is mainly based on a clinical examination [5]. Dermatoscope is useful to diagnose wart lesions through the description of their distinctive dermoscopic patterns and vascular features [6]. There are several modalities to diagnose AGW, including histopathology, confirmatory PCR method to detect HPV DNA, and analysis of HPV genotype as a definitive diagnosis $[7,8]$.

Furthermore, the treatment of AGW is challenging. Among available medical and destructive therapeutic options, none is uniformly effective. Many virucidal topical treatments achieve clearance rates ranging only from 35 to $81 \%$ [7]. Also, side effects of any therapy include local skin reactions, such as pain, itching, burning, skin erosions, and scarring [6]. Therefore, there is an increasing demand for herbal medicines as an effective, affordable, user-friendly, and alternative therapies to treat AGW. Glycyrrhizinic acid (GA) is a substance found in Glycyrrhiza glabra root, commonly known as licorice. It has been previously studied for AGW treatment among immunocompetent Caucasian patients, with high satisfactory result $[7,9]$; although, studies with immunocompromised patients have not been performed. In order to fill this gap in literature, and to find the most effective medication for AGW among immunocompromised patients, we conducted this case series. The aim was to evaluate the response of GA $0.1 \%$ spray as an alternative treatment for AGW among HIV-positive Asian patients in Indonesia.

\section{Cases description}

We reported two cases of AGW from outpatient clinic of dermato-venerology department, Dr. Hasan Sadikin General Hospital, Bandung. All the patients' lesions were monitored, and their appearances recorded.

\section{Case 1}

A 30-year-old Chinese-Indonesian female patient came to our clinic with asymptomatic, multiple papules for three

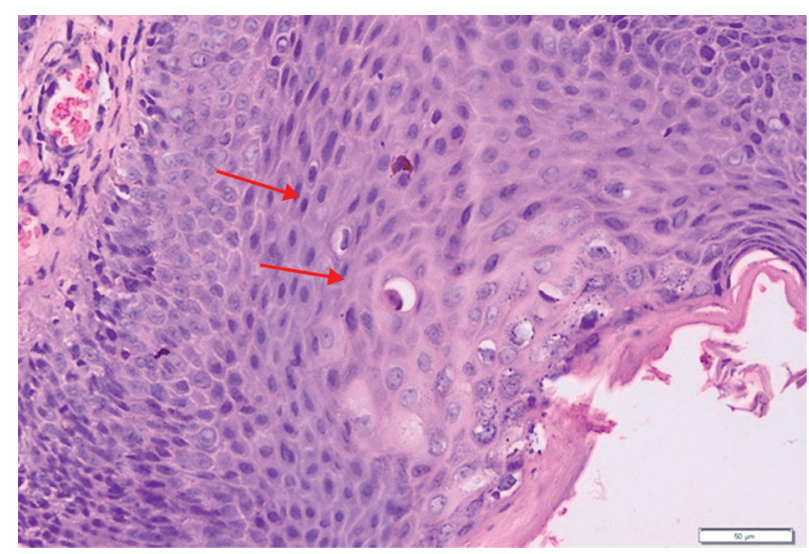

Figure 1. Histopathology showing epidermal hyperplasia with koilocytes H\&E (200x) months. She had been HIV-positive for ten years and on HAART (zidovudine, lamivudine, and nevirapine) over the last 6 months. The latest CD4 cell count was 100 cells/ $\mu \mathrm{l}$ (normal values, 404-1,612 cells/ $\mu \mathrm{l}$ ). Physical examination (PE) revealed multiple, discrete, 1-2 mm skin-colored to brownish papules, some with flat surface, symmetrically distributed throughout the mons pubis, inner sides of labia majora and minora. A shave biopsy was performed, and histopathology examination confirmed a diagnosis of condyloma acuminatum (CA) (Figure 1). PCR examination was performed, revealing type $6 \mathrm{HPV}$ DNA. Dermoscopic examination revealed finger-like pattern with dotted and glomerular vessels (Figure 2A). The patient was treated with GA $0.1 \%$ spray, three times a day for twelve weeks. The medication was applied by the patient herself. In the second week of the treatment, some parts of the lesion disappeared. Dermoscopy results showed the lesion to evolve into knob-like pattern (Figure 2B). In the twelfth week, there was a slight lesion improvement; some parts of the lesion remain unchanged and some parts of persistent lesion were noted (Figure 3). Therefore, we changed the treatment.

\section{Case 2}

A 27-year-old male Bornean MSM came to our OPD. The patient was suffering from perianal wart for three months, which gradually increased in size. Physical examination revealed multiple, fleshed-colored, moist papules and plaques, with verrucous surfaces on the perianal area. Anal examination showed that the anal canal was not infected. The patient had been diagnosed with HIV for a month and was taking antiretroviral therapy (lopinavir/ritonavir, lamivudine, and nevirapine). Laboratory analysis showed CD4 value of 348 cells/ $\mu$ l, and histopathological examination confirmed the diagnosis of CA. PCR examination revealed type 6 and $11 \mathrm{HPV}$ DNA. Dermatoscope examination showed finger-like pattern, with glomerular and hairpin vessels (Figure 4A). Similar to the previous patient, we suggested the application of GA $0.1 \%$ three times a day for eight weeks. In the second week, the lesion reduced in size and the surface flattened and was less verrucous. Dermoscopy result revealed that the lesion evolved into knob-like pattern with thrombosed vessels (Figure 4B). During the twelfth week, the size decreased even more, and lesions in few location started to disappear, but some parts of the lesion remain unchanged (Figure 5).

\section{Discussion}

Dermoscopy is a non-invasive, simple, and inexpensive method of observation of morphological characteristics of skin surface and lower epidermis in vivo, which are invisible to naked eyes [8]. Besides having an important value for diagnosing skin diseases, it can be used for monitoring treatment response $[10,11]$. The dermoscopic patterns of AGW are finger-like, knob-like, or mosaic pattern along with vascular structures [11]. The vascular features consist of hair- 

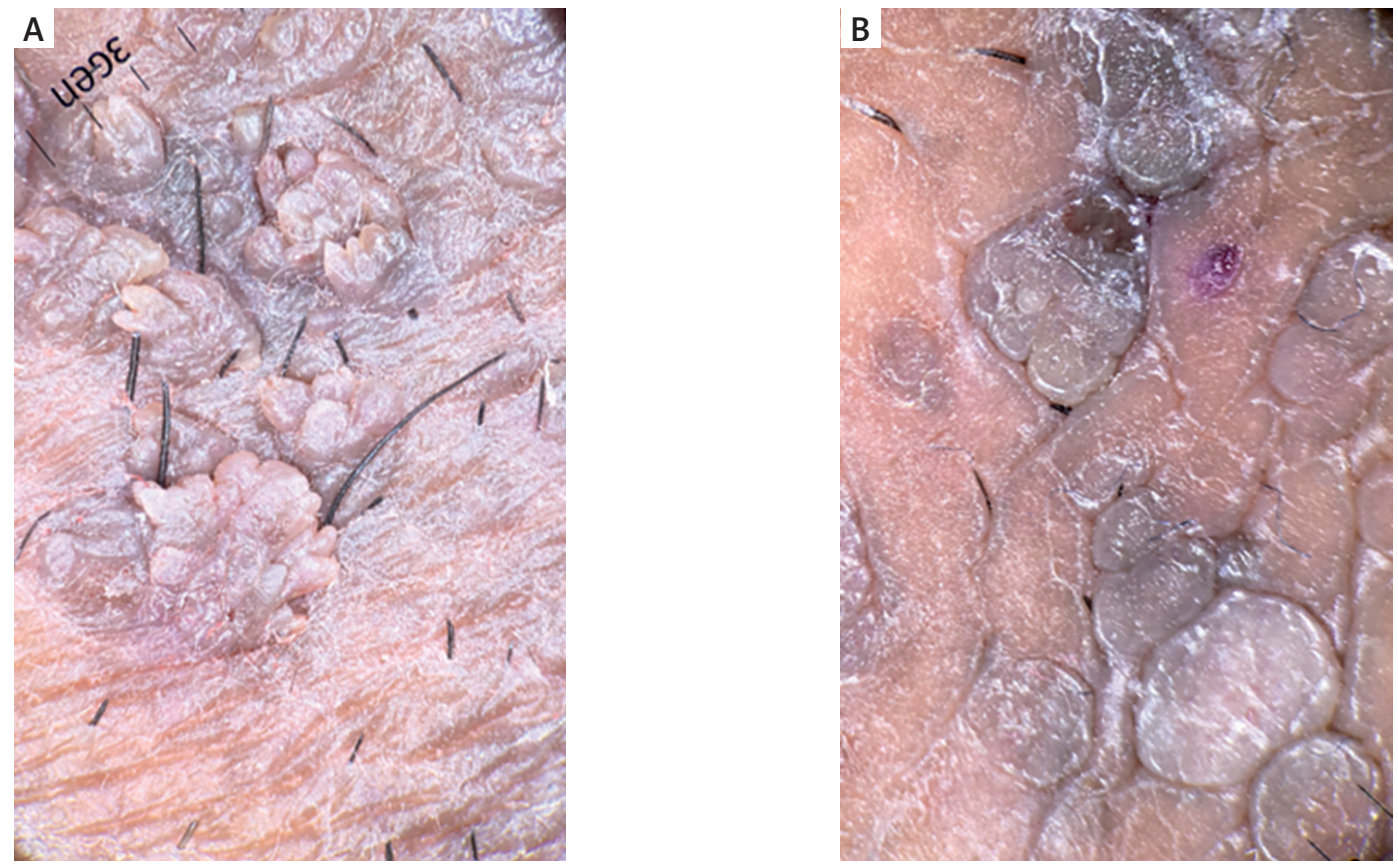

Figure 2. A) Anogenital wart with finger-like pattern and glomerular vessels (before treatment). B) At 12-week, knob-like pattern with flattening of some parts of the lesion was noted
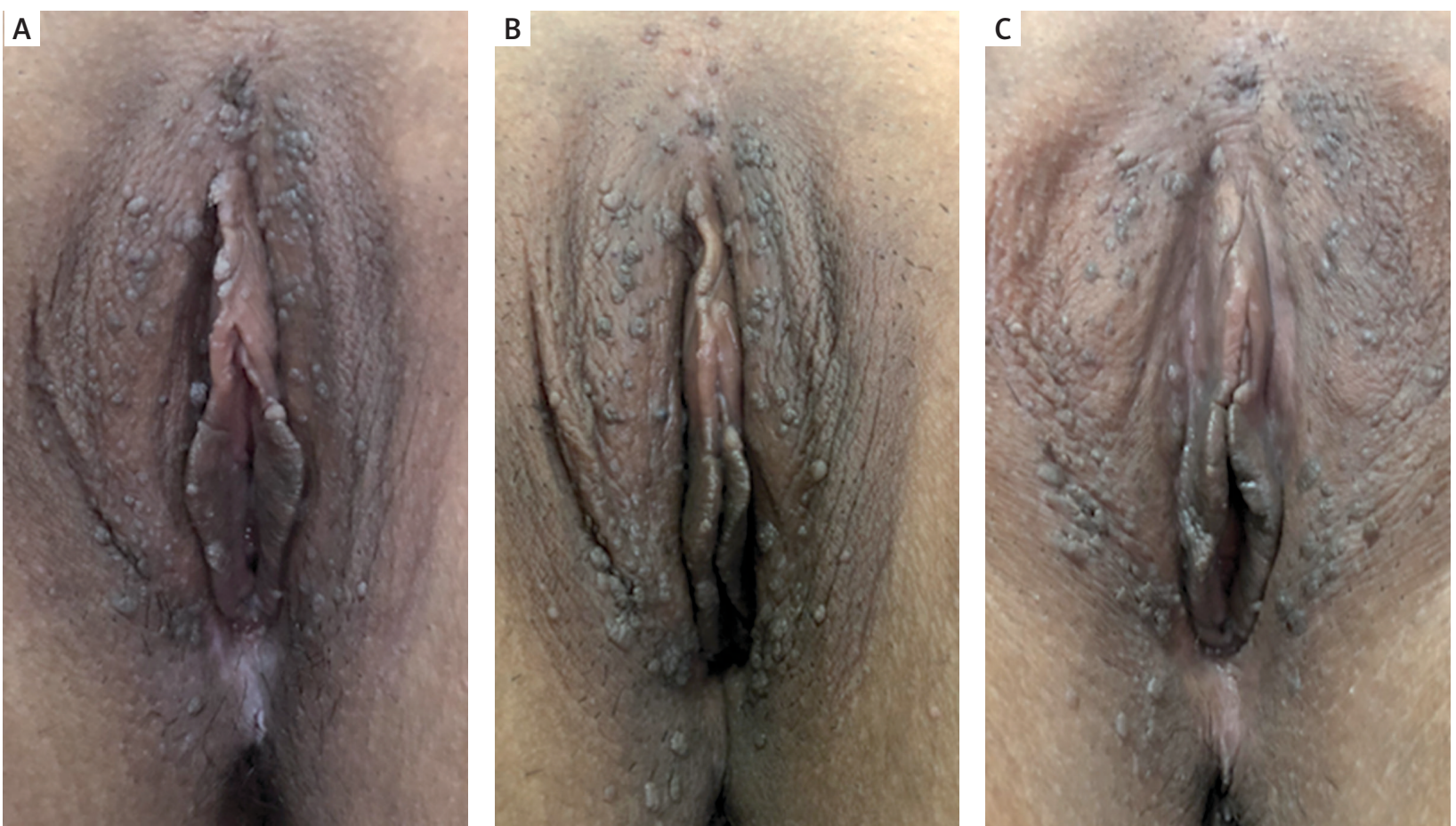

Figure 3. A-B) After two weeks of treatment, some parts of the lesion disappeared. C) After twelve weeks, persistence of some parts of the lesion was observed

pin, glomerular, or dotted vessels. The dermoscopic pattern may be found useful in monitoring the efficacy of treatment of viral warts [12]. In both of our cases, the appearance of finger-like pattern evolving to knob-like pattern after six weeks of treatment, showed a positive good response to the GA $0.1 \%$ spray treatment. 

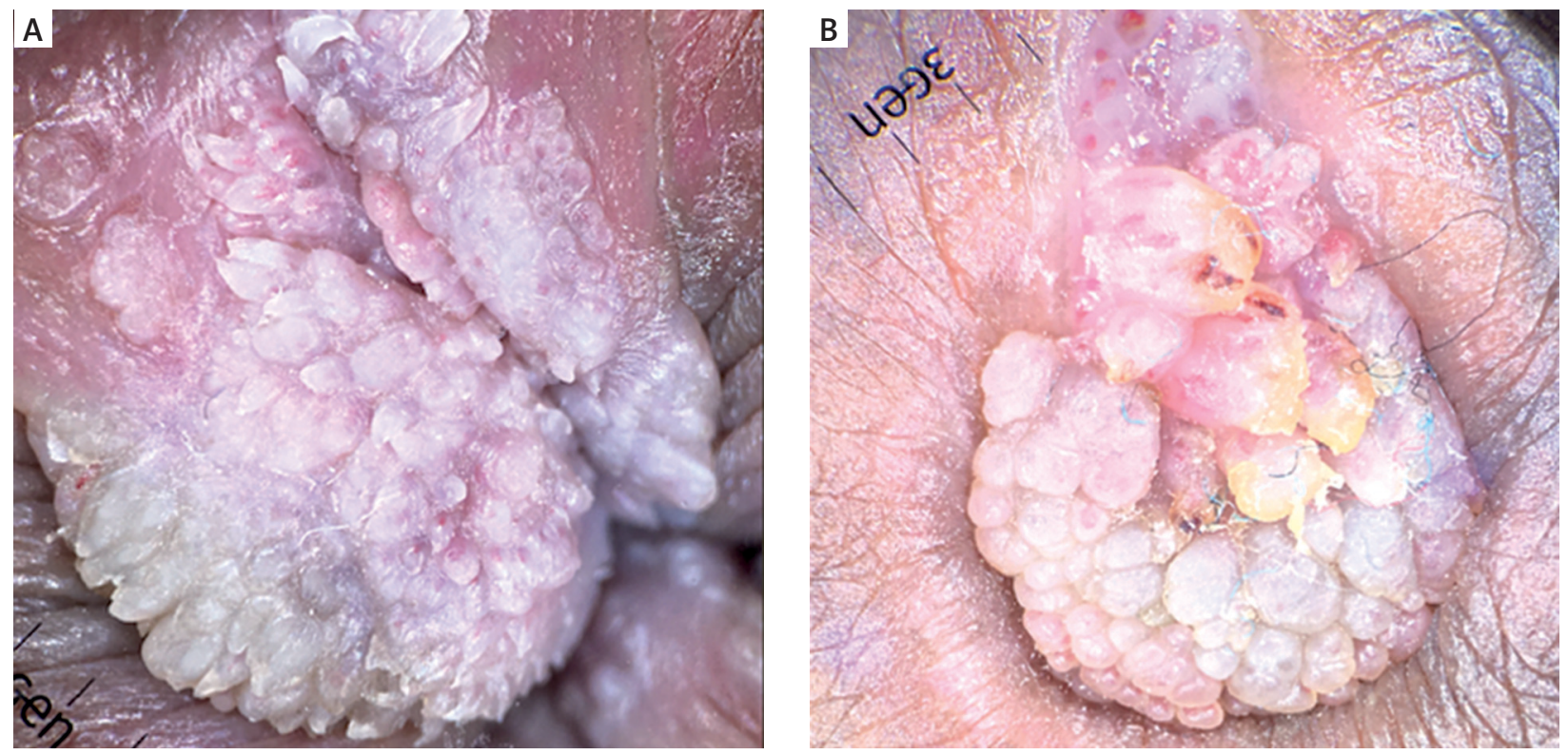

Figure 4. A) Anogenital wart with finger-like pattern and hairpin vessels (before treatment). B) At 12-week, knob-like pattern with flattening in some parts of the lesion and thrombosed vessels were observed
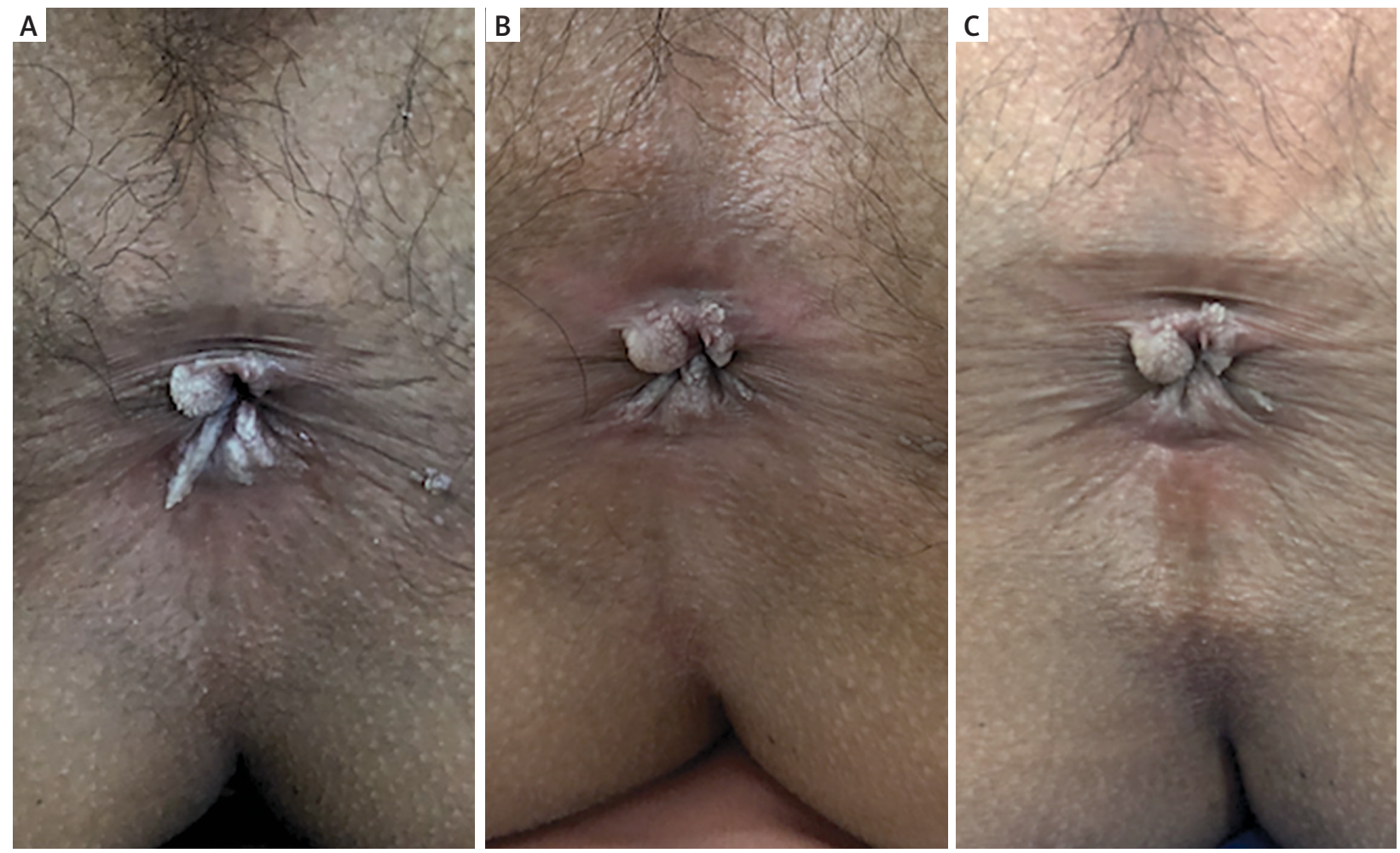

Figure 5. A-B) After two weeks of treatment, parts of the lesion were reduced in size and the surface was flatten and less verrucous. C) On twelfth week, a slight reduction in size in parts of the lesion was observed

Histopathologically, the hallmark of HPV-infected cell is the development of morphologically atypical keratinocytes called "koilocytes" [12]. These are enlarged cells with eccen- tric, pyknotic nuclei that are often surrounded by a perinuclear halo [12]. In the present study, the appearance of koilocytes were observed in both cases. PCR is a highly specific 
and sensitive test for HPV genotyping [13]. Here, the cases diagnosed under dermoscopy were confirmed by histopathology and HPV DNA typing of the patients.

Regarding the treatment, biological actions of GA have been widely studied. The efficacy and tolerability of GA $0.1 \%$ spray have previously been assessed in few studies in immunocompetent patients $[12,13]$. Overall results were promising, with reported improvement rates between $68.4 \%$ and $87.5 \%$ [7, 12]. Gomez et al. treated 100 immunocompetent AGW patients with GA $0.1 \%$ spray versus podophyllin $25 \%$ solution for eight weeks [6]. They reported that GA $0.1 \%$ spray had an $87.5 \%$ efficacy rate, which was slightly better than podophyllin [6]. Additionally, Gomez et al. argued that papillomatous lesions responded better to GA treatment, and there was no adverse reaction observed [6]. A randomized, double-blind controlled study with 76 AGW pediatric patients conducted by Romo et al. revealed that topical treatment of GA $0.1 \%$ spray, applied three times a day for twelve weeks, resulted in $68.4 \%$ of complete remission and $28.9 \%$ of decrease in lesion by more than $75 \%$ [10]. In terms of structure, GA is a type of triterpene saponin that contains hydrophobic region (one molecule of glycyrrhetinic acid). GA has an advantage over other topical regiments due to its amphipathic and solubilizing properties; therefore, it could achieve a higher deposition and penetration rate in the epidermal layer [14]. GA stimulates production of IL-12 in macrophages and this facilitates the development of T helper lymphocytes in cell-mediated immune response, inhibits viral protein phosphorylation mediated by cellular kinases, and modifies post-translational signals essential for growth [12]. GA also could induce IFN production, which promotes the activation of macrophages and phagocytes, and destruct microorganisms [13]. Research has discovered its role in the production of gamma interferon (IFN- $\gamma$ ), which reduces tumor necrosis factor and secretion of interleukin 1 (IL-1) as well as its role in stimulating IL-10 [11]. GA increases the production of viral cyclin that induces selective death of cells infected by virus $[11,14]$.

In both of our patients, the treatment resulted in poor response, with only slight reduction in size and number in some parts of the lesion after twelve weeks of treatment. The reason was that in HIV infection, CD4+ T cells that play a major role in protective response against microorganism including the virus, have already been infected and destroyed by HIV. Although the amount of CD4 were different in both cases, they were both below normal range. This low number of CD4 may explain poor responses in both cases. Further research need to be performed to evaluate a correlation of CD4 level with a response of GA treatment in AGW patients.

\section{Conclusions}

Our case series showed that GA $0.1 \%$ was only partially efficacious for AGW among HIV-positive patients. To our knowledge, there is no published study in the literature describing GA as a treatment for immunocompromised patients evaluated clinically and dermoscopically. Dermoscopy is useful in the evaluation of treatment progress, as it can accurately record morphology lesion of AGW. Further studies should be performed in order to investigate a relation between cellular immune response represented by the number of CD4 with GA treatment response in AGW patients.

\section{Disclosure}

The authors have no potential conflicts of interest to disclose.

\section{References}

1. Patel H, Wagner M, Singhal P, Kothari S. Systematic review of the incidence and prevalence of genital warts. BMC Infect Dis 2013; 13: 1-14.

2. Cusini M, Salmaso F, Zerboni R, et al. $5 \%$ Imiquimod cream for external anogenital warts in HIV-infected patients under HAART therapy. Int J STD AIDS 2004; 15: 17-20.

3. Bilenchi R, Campoli M, Trovato E, Cinotti E, Rubegni P, Fimiani M. Sinecatechins $10 \%$ ointment for genital warts: case report of a beneficial reaction in an HIV-positive woman. Int J STD AIDS 2018; 29: 1033-1035.

4. Lacey CJ, Woodhall SC. European guideline for the management of anogenital warts. J Eur Acad Dermatol Venereol 2013; 27: 263-270.

5. Dong H, Shu D, Campbell TM, Fruhauf J, Soyer P, Wellenhof R. Dermatoscopy of genital warts. J Am Acad Dermatol 2011; 64: 859864.

6. Gómez JG, Simón RD, Daniel AA, Zelenkova H. Effectiveness of glycyrrhizinic acid (Glizigen) and an immunostimulant (Viusid) to treat anogenital warts. ISRN Dermatol 2012; 2012: 863692.

7. Stanley MA. Genital human papillomavirus infections: current and prospective therapies. J Gen Virol 2012; 93: 681-691.

8. Zhang Y, Jiang S, Lin H, Guo X. Application of dermoscopy image analysis technique in diagnosing urethral condylomata acuminata. An Bras Dermatol 2018; 93: 67-71.

9. Zalaudek I. Dermoscopy in general dermatology. In: Marghoob AA, Broun R (eds.). An Atlas of Dermoscopy. 2 ed. London: Informa Health; 2012: 333-337.

10. Romo EM, Fundora FP, Ramirez CR, Rodriguez AY, Hana Z. Effectiveness of glycyrrhizinic acid and immunostimulant to treat genital warts in the pediatric patient population. Innovative J Med Health Sci 2013: 3: 88-93.

11. Zelenkova H, Nejdkova A, Skutilova E, Urbani M, Svecova D, Cabalova J. Preparations containing glycyrrhizic acid employed in dermatovenereologic practice. Conclusions of an international multicentre study. Dermatol Klin 2005; 7: 135-139.

12. Wang Y, Zhao B, Wang S, et al. Formulation and evaluation of novel glycyrrhizic acid micelles for transdermal delivery of podophyllotoxin. Drug Deliv 2016; 23: 1623-1635.

13. Valencia MH, Pacheco AC, Quijano TH, Girón AV, López CV. Clinical response to glycyrrhizinic acid in genital infection due to human papillomavirus and low-grade squamous intraepithelial lesion. J Clin Pract 2011; 93: 200-202.

14. Sandy OC, Rios RF, Cedillo CM, et al. Focal multiepithelial hyperplasia: comparative treatment, glycyrrhizinic acid versus liquid nitrogen. Revista Odontológica Mexicana 2015; 19: 101-105.

15. Vijayan KKV, Karthigeyan KP, Tripathi SP, Hanna LE. Pathophysiology of CD4+ T-cell depletion in HIV-1 and HIV-2 infections. Front Immunol 2017; 8: 580-588. 